\title{
On the validation of integrated DIC with tapered double cantilever beam tests
}

\author{
Thiago Melo Grabois ${ }^{\mathrm{a}, \mathrm{c}, *}$, Jan Neggers ${ }^{\mathrm{b}}$, Laurent Ponson ${ }^{\mathrm{c}}$, François Hild ${ }^{\mathrm{b}}$, \\ and Romildo Dias Toledo Filho ${ }^{\mathrm{a}}$ \\ ${ }^{a}$ Laboratory of Structures and Materials Professor Lobo Carneiro (LabEST), COPPE / \\ UFRJ, P.O. Box 68506, Zip code 21941-972, Rio de Janeiro, Brazil \\ ${ }^{b}$ Laboratoire de Mécanique et Technologie (LMT), ENS Paris-Saclay / CNRS / \\ University Paris-Saclay, 61 Avenue du Président Wilson, 94235 Cachan Cedex, France \\ ${ }^{c}$ Institut Jean le Rond d'Alembert, UPMC - CNRS, Sorbonne Universités \\ 4 Place Jussieu, 75005 Paris, France
}

\begin{abstract}
Full-field measurement methods such as integrated digital image correlation bypass the need for post-processing the displacement data upon minimizing the gap between simulation and experiment. This paper discusses the validation of this method by comparing its results with an approach based on compliance values measured from crack mouth opening displacement and load data to calculate mode I stress intensity factors (SIFs) and crack tip positions. Tapered double cantilever beams are used as a test case to evaluate the quasi-brittle fracture response of PMMA. The results demonstrate the ability of the proposed technique to obtain accurate determinations of SIFs and crack length using the tapered geometry. Additionally, both methods yield consistent results, which validates the integrated approach against an independent method.
\end{abstract}

\footnotetext{
*corresponding author

Email address: thiagomgrabois@coc.ufrj.br (Thiago Melo Grabois)
} 
Keywords: Compliance method, Digital image correlation, Stress intensity factor measurement, Stable crack propagation

\section{Introduction}

Accurately measuring the material toughness is a difficult task for several reasons. First, the toughness is defined as the critical driving force (or critical elastic energy release rate) at which a crack propagates. In principle, this means that both the crack tip position and the total energy released during crack advance must be tracked during the test, thereby rendering the measurement very indirect and prone to multiple causes of experimental errors. Second, the local toughness value, namely $K_{I c}$ may depend on the crack growth velocity $v$. These facts require the development of fracture tests where the crack propagation speed can be controlled and tuned over a broad range. Furthermore, measuring toughness requires stable fracture, both from the point of view of crack propagation (i.e., the crack must stop when the external driving force is stopped) and trajectory that must be rather straight. This can be particularly challenging in brittle solids (e.g., ceramics and glass). The choice of the geometry of the fracture test that controls the crack stability then becomes essential.

Different fracture tests have been proposed to handle these challenges. Some of them, such as Single Edge Notch Bending (SENB) and Compact Tension (CT) tests [1], present a short crack propagation length and a rather poor stability of the fracture process [2]. Alternatively, the Tapered Double Cantilever Beam (TDCB) test enables for significant stability of the fracture process in mode I [3], which is appealing for brittle materials. Further, it 
allows for the exploration of a large range of crack growth velocities $[4,5]$. Different TDCB specimens were used to perform mode I fracture tests of adhesive bond joints $[6,7,8]$ and, for instance, to study the R-curve behavior in quasi-brittle wood materials [9] taking advantage of the crack growth stability provided by the tapered shape. In many displacement-controlled TDCB experiments, it has been observed that the compliance increases linearly with the crack length. Moreover, previous studies [8, 10, 11] supported this fact using beam theory. As the energy release rate [6] depends on the derivative of the compliance, its value is then constant when the crack propagates.

Recently, the TDCB geometry was modified by significantly enlarging the arms from which the displacement is prescribed during the test $[4,5]$. This new design results in an exponential decrease of the elastic energy release rate with crack length; and thus in an improved stability of the fracture process, thereby rendering the TDCB fracture test amenable to the study of brittle solids.

Standardized test methods for measuring fracture toughness usually consider global measurements provided by load cells and clip gauges (e.g., see Ref. [1]). An alternative route consists in utilizing full-field measurements. Digital Image Correlation (DIC) was successfully used to monitor cracks in various materials $[12,13,14,15,16]$. To determine Stress Intensity Factors (SIFs) and crack tip positions, the measured displacement fields are subsequently post-processed via projections onto known solutions of fractured elastic media [12]. In the case of brittle fracture, it is assumed that, apart from the immediate vicinity of the crack tip, the material behaves elastically. In this specific case, Williams' series [17] describe the stress and displacement 
fields as functions of mechanical parameters for different fracture modes. A least-squares fit enables the SIF to be evaluated $[12,13,14]$ provided the crack tip position is known [18]. Dehnavi et al. [19] showed that this method may provide more accurate results compared to photoelasticity when evaluating SIFs.

The aim of the present paper is to validate Integrated DIC [18, 20]. Instead of first measuring displacement fields and then projecting them onto ${ }_{55}$ Williams' series, the two steps are integrated into a single analysis where the sought amplitudes (e.g., SIFs, crack tip positions) are a direct result of the registration method. This approach bypasses the need for post-processing the displacement data, thereby reducing errors in the re-projection process [16]. This is of particular importance in the present case since the displacements are very small [21]. The crack tip location and SIFs will be compared with analyses based upon compliance measurements obtained independently from crack mouth opening displacement (CMOD) and load data.

The paper is organized as follows. First the experimental configuration and the studied material are introduced. The procedure employed to measure the compliance variations during crack growth that will be used herein as a validation tool is presented. The integrated DIC framework is then summarized in order to follow crack propagation and estimate SIFs. The procedure is then applied to the pictures of two TDCB tests and the measurements are compared with the results of the compliance technique discussed herein. 


\section{TDCB fracture tests}

Experiments on Tapered Double Cantilever Beam (TDCB) specimens were carried out under uniaxial tension using a Shimadzu (model AG-Xplus) universal testing machine (with $\pm 10 \mathrm{kN}$ load capacity). In this experimental setup (see Figure 1(a)), a $1 \mathrm{kN}$ load cell measured the force applied to the specimen through the pins located in holes of diameter $2 R=5 \mathrm{~mm}$. For the first experiment, a clip gauge was used to measure the crack mouth opening displacement. Two steel grips were connected to the specimen by the pins placed on both holes. The bottom one is fixed to the base and the top one, which connects to the load sensor, is pushed up by the machine cross-head.

80 namely, poly-methyl methacrylate (PMMA), which is an archetype of brittle amorphous material [22]. Two TDCB specimens were cast from a thin PMMA sheet of $8 \mathrm{~mm}$ thickness using a laser cutting machine. The specimen geometry is an isosceles trapezoid of shorter base $h_{1}=60 \mathrm{~mm}$, longer base in length extends from the midpoint of $h_{1}$. Although the main TDCB geometry is the same for both experiments reported herein, they were managed differently. Thus, the following procedures are discussed in this work:

(i) The first experiment was controlled by the crack mouth opening rate at
$5 \mu \mathrm{m} / \mathrm{s}$, i.e., with the clip gauge opening at constant velocity. In this case, the specimen $V$ shaped notch experienced a straight elongation of $3.5 \mathrm{~mm}$ through laser cutting and an additional $20 \mathrm{~mm}$ crack extension achieved by pre-loading the specimen under constant (i.e., $0.5 \mu \mathrm{m} / \mathrm{s}$ ) crack mouth opening velocity until the crack initiated. The sample 


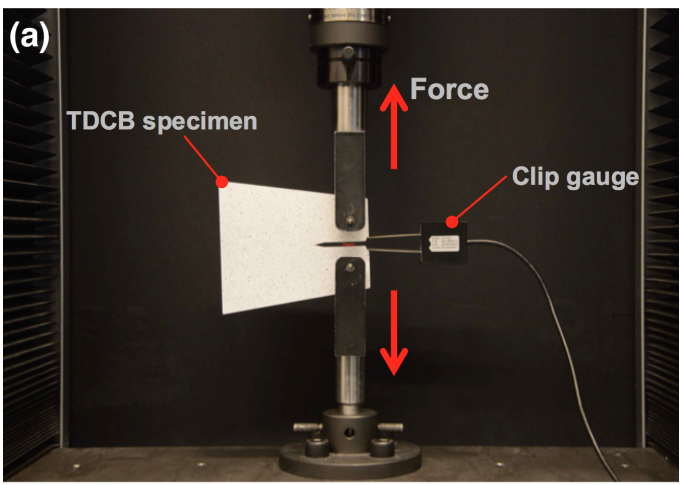

(a)

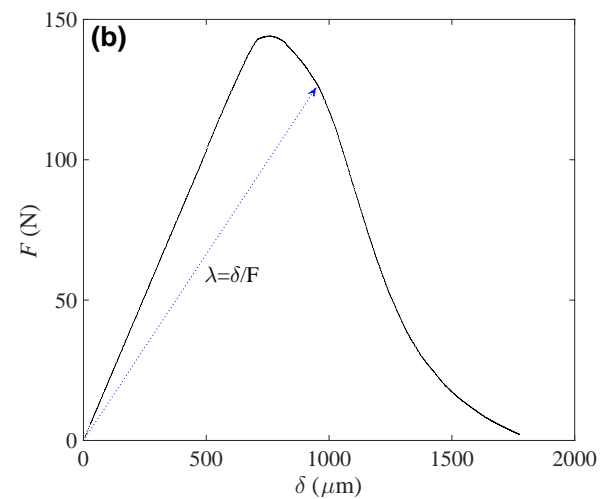

(b)

Figure 1: (a) Experimental test setup used to perform quasi-static TDCB tests. The electromechanical machine was equipped with a load cell and a clip gauge to measure the applied force and crack mouth opening displacement, respectively. (b) Typical forcedisplacement curve for a TDCB experiment of a PMMA specimen of initial crack length $c_{i n i}=60 \mathrm{~mm}$ submitted to a CMOD rate of $5 \mu \mathrm{m} / \mathrm{s}$. 
fracture experiments are presented. First, a finite element (FE) based method is employed to assess the elastic energy release rate and the crack speed from the macroscopic mechanical behavior of the specimen, namely, the forcecrack opening response, an example of which is presented in Figure 1(b). Then, it is shown that the TDCB geometry is amenable to a direct determination of SIFs through DIC. This is an interesting alternative to the prior methodology as it allows the fracture properties of the tested material to be estimated without using load cells and CMOD gauges. In particular, such alternative may be useful to measure toughnesses of high temperatures or for high crack speeds for which mechanical gauges may be inefficient. Moreover, the DIC method provides the mode II stress intensity factor (in addition to $K_{I}$ ) that is of relevance for anisotropic solids as well as the amplitude of non-singular terms such as the T-stress not accessible through the analysis of the force-displacement response of the specimen.

\section{Compliance method}

To implement this first method, only the force and crack opening displacement data are considered. The goal is to determine the material fracture toughness $K_{I c}$ and crack velocity $v$ histories during the TDCB test. The experimentally measured quantities are post-processed to determine the fracture properties via finite element simulations. The flowchart shown in Figure 2 illustrates the procedure. The experimental compliance $\lambda_{\exp }=\frac{\delta}{F}$ is compared with the finite element prediction $\lambda_{\mathrm{FE}}(c)$ to infer the crack length, since a bijective relationship exists between these two quantities as shown in the following. Then, from the measured force and crack length, the elastic 
130

energy release rate $G=F^{2} g_{\mathrm{FE}}(c)$ follows. The crack velocity is computed as the time derivative of the crack positions. The objective of the finite element simulations is thus to extract $\lambda_{\mathrm{FE}}(c)$ and $g_{\mathrm{FE}}(c)$ that are geometry-dependent functions.

This procedure is inspired from the compliance method used for instance 135 fied TDCB geometry used in this work $[4,5]$. The method is briefly described in the sequel. It is based on the following two assumptions: i) brittle fracture i.e., the material behaves elastically, except in a zone around the crack tip, which is very small with respect to the sample size; ii) time-independent 140 behavior (e.g., viscosity is neglected). Numerical investigations of the mechanical response of modified TDCB specimens are detailed in Refs. [4, 5]. 


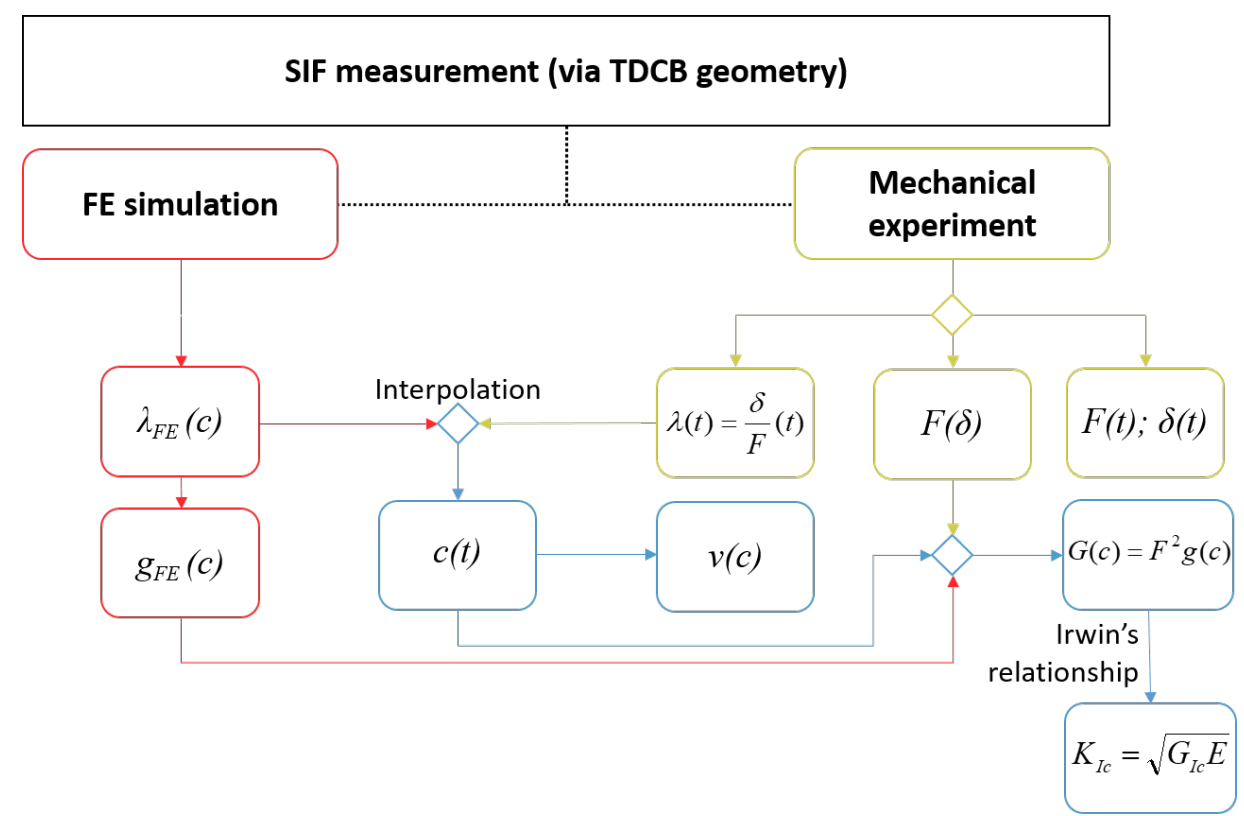

Figure 2: Overview of the proposed SIF measurement procedure based on the analysis of the macroscopic mechanical response of the specimen. In red: $\lambda_{\mathrm{FE}}$ and $g_{\mathrm{FE}}$ are the compliance and the elastic energy release rate functions determined by FE simulations. In yellow: the experimental force, displacement, time, and compliance $(F, \delta, t$, and $\lambda$, respectively). In blue: the crack length $c$, crack velocity $v=\frac{d c}{d t}$, the energy release rate $G$, and the fracture toughness $K_{I c}$ determined after coupling experimental data with $\mathrm{FE}$ simulations.

2D plane stress finite element calculations were performed using the open source package Cast3M [25]. The simulations are based on the solution to a linear elastic problem at fixed crack position. The crack length $c$ is varied incrementally with steps $\delta c=0.005 L$ to obtain the variations of the sought quantities. Based on the sample symmetry, the analysis is limited to the upper half of the TDCB geometry. The mesh size decreases exponentially while approaching the crack tip, down to a minimum element size of 
$10^{-9} \mathrm{~L}$. For each crack tip position, the compliance is obtained by prescribing a unit force $F_{0}$ and computing the corresponding displacement $\delta$ so that $\lambda_{\mathrm{FE}}=\delta / F_{0}$. The elastic energy release rate is obtained from the variation of mechanical energy for an incremental advance of the crack. The computed value was confirmed through an independent method based on the so-called compliance formula (see Equation (3)). The finite element solution for the non-dimensional compliance $\lambda E b$, where $E$ is the material Young's modulus and $b$ the specimen thickness, is shown in Figure 3. For the range of crack lengths $L / 3 \lesssim c \lesssim 2 L / 3$, the compliance can be approximated by an exponential function

$$
\lambda=\frac{\lambda_{0}}{E b} \mathrm{e}^{c / c_{0}}
$$

where $\lambda_{0}=6.0$ and $c_{0}=21 \mathrm{~mm}$ are fitting parameters. When the crack is very close to the boundary, the compliance deviates from the exponential approximation. As shown in Figure 3, this behavior is confirmed experimentally by measuring the specimen compliance for different crack lengths in a cyclic TDCB experiment using PMMA $[4,5]$. 


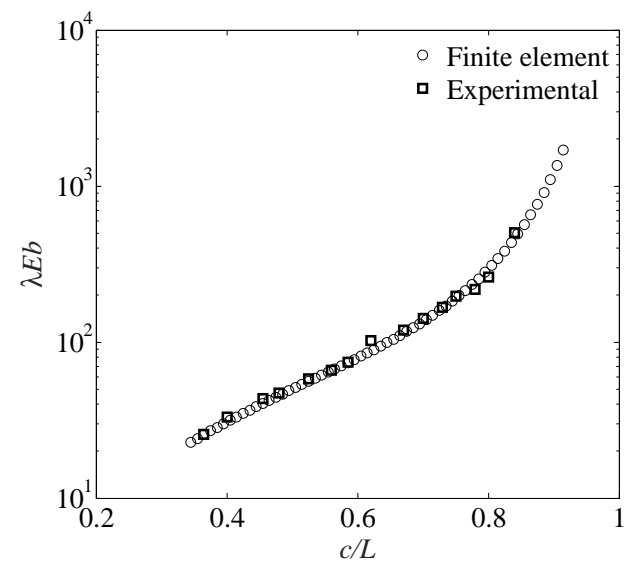

Figure 3: Variation of the normalized compliance with crack length for the TDCB specimen. The open circles are FE results while the open squares are data from a cyclic experiment using a PMMA specimen with $b=8 \mathrm{~mm}$ and $E=2.3 \mathrm{GPa}[4,5]$.

Interestingly, the variations of the normalized elastic energy release rate 165 $g(c)$ can be derived from Equation (1) using the so-called Irwin-Kies equation [26], also known as the compliance formula ${ }^{1}$

$$
g=\frac{1}{2 b} \frac{d \lambda_{F}}{d c}
$$

that leads to

$$
g=\frac{r_{0} \lambda_{0}}{2 E b^{2} c_{0}} .
$$

It is therefore possible to obtain the elastic energy release rate as a function of crack length $G(c)$, once the compliance has been computed as a function of crack length (see e.g., Figure 3). The combination of Equations (1)

\footnotetext{
${ }^{1}$ Strictly speaking, the Irwin-Kies formula applies to the compliance $\lambda_{F}$ defined from the displacement at the point of application of the force, and not from the CMOD. However, $\lambda_{\mathrm{F}}$ and $\lambda$ are found to be proportional, irrespective of the crack length, leading to $\lambda_{\mathrm{F}}=r_{0} \lambda$ where $r_{0} \simeq 0.80$ is obtained from FE calculations.
} 
and (3) provides the elastic energy release rate $G=F^{2} g(c)=\frac{\delta^{2}}{\lambda(c)^{2}} g(c)$ under displacement controlled conditions, leading to

$$
G(c)=\delta^{2} \frac{r_{0} E}{2 \lambda_{0} c_{0}} e^{-c / c 0}
$$

The exponential decay of $G$ with crack length points out the high stability of the fracture process of the modified TDCB geometry for displacement175 controlled loading conditions. Last, the mode I SIF derives from Irwin's relationship [27] under plane stress condition

$$
K_{I}=\sqrt{G E}
$$

In practice, the experimental compliance $\lambda_{\exp }(t)=\delta / F$ measured at each time step is compared with the compliance $\lambda_{\mathrm{FE}}$ computed numerically, leading to $c(t)$ as illustrated in Figure 4. For instance, the experimentally measured value of $\lambda_{\exp }^{*}$ at some time $t^{*}$ (lower right inset) provides the crack length $c^{\star}\left(t^{\star}\right)$ through the comparison with the finite element prediction $\lambda_{\mathrm{FE}}(c)$ (upper left inset). For experiment \#2 that does not use clip gauge, the CMOD is computed after the test using DIC by measuring the displacement $\delta(t)=u_{B}(t)-u_{A}(t)$ of point $\mathrm{B}$ with respect to point $\mathrm{A}$, where $\mathrm{A}$ and $\mathrm{B}$ are the material points where the clip gauge is placed in experiment $\# 1$. Thus, the very same procedure based on the compliance measurement can be used for both tests. 


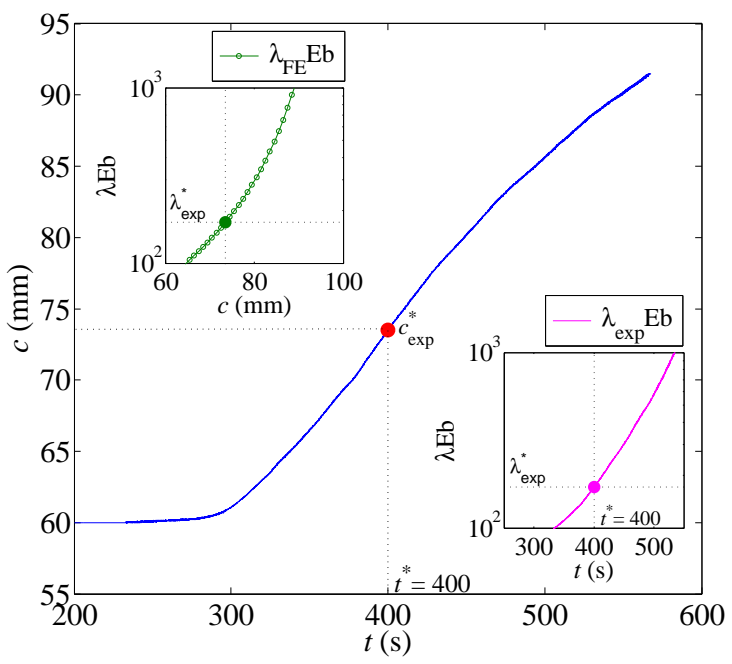

Figure 4: Method used to determine the crack length and its variations as a function of time. The bottom inset shows the experimentally measured compliance $\lambda_{\exp }$ normalized by $E b$ as a function of time. For a given time (e.g., $\left.t^{*}=400 \mathrm{~s}\right)$, the corresponding $\lambda_{\exp }^{*}$ is highlighted by the solid circle. The top inset shows the finite element results for the variations of the compliance $\lambda_{\mathrm{FE}}$ normalized by $E b$ as a function of crack length $c$. The comparison with $\lambda_{\exp }^{*}$ provides the crack length $c_{\exp }^{*}$. The main panel shows the obtained crack length $c_{e x p}$ as a function of time. The solid red circle corresponds to $t^{*}$ and $c_{\exp }^{*}$.

\section{Digital image correlation}

Digital Image Correlation (DIC) has been successfully used for various

190

applications in experimental mechanics [28, 29, 30] since the 1980's. In the present case, the analyses will be based upon 2D images shot by a single camera (i.e., 2D-DIC).

\subsection{Imaging acquisition}

Picture acquisitions were carried out with one CMOS camera (Baumer HXC20 digital monochrome full progressive scan camera, definition: $1088 \times$ 
2048 pixels). A Zeiss fixed lens (100 mm F2, model Makro-planar T*) and a Nikon extension tube (52.5 mm, PN-11 model) were used. The pictures were acquired with a frame rate of $1 \mathrm{~Hz}$. Figure 5 shows the reference image of the tested specimen surface where a random black and white speckle pattern was sprayed. The working distance is about $70 \mathrm{~cm}$ and the pixel size corresponds to $48 \mu \mathrm{m}$ with this magnification. The region of interest (ROI) covers part of the image whose size is $1080 \times 2040$ pixels with 8 -bit digitization. This image histogram (Figure 5 (right)) covers the whole dynamic range of 256 gray levels.
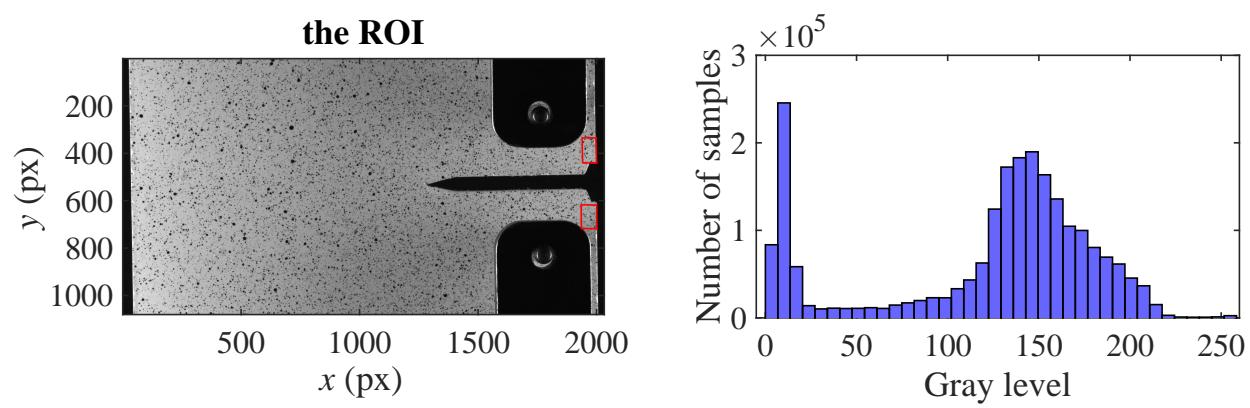

Figure 5: Reference picture of the specimen surface (left) and corresponding gray level histogram (right). The boundary of the two regions of interest (ROI) for the T3-DIC displacement measurement (substituting the clip gauge measurements) are shown as red boxes.

A T3-DIC analysis (with an unstructured mesh made of 3-noded elements) with 42-pixel mean length is first performed. The displacement fields in both directions are displayed in Figure 6 for picture no. 239 (from a total of 380 for experiment \#2) corresponding to the first one after crack initiation. The discontinuity of the field is clearly evidenced for the $y$ displacement component corresponding to the loading (i.e., vertical) direction. Note that 
the highest amplitude of the displacements in this direction is about 4 pixels (i.e., $\approx 200 \mu \mathrm{m}$ ).

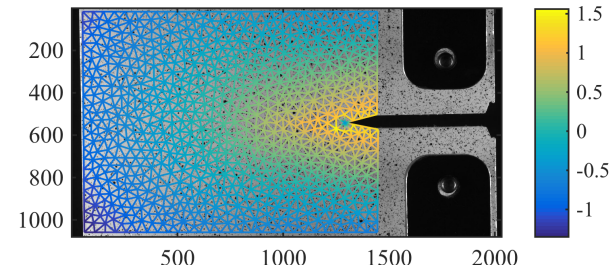

(a)

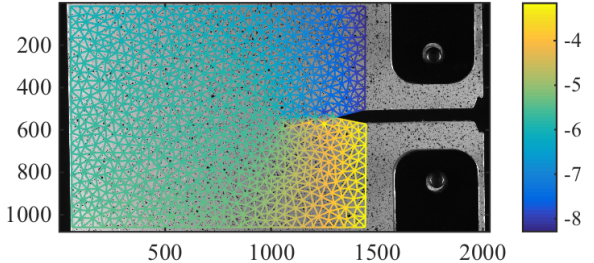

(b)

Figure 6: Displacement fields just after crack initiation (i.e., image 239 or $t=239 \mathrm{~s}$ ) for the second TDCB test. Horizontal $u_{x}$ (a) and Vertical $u_{y}$ (b) displacement maps using T3-DIC. The values are expressed in pixels (1 pixel $\equiv 48 \mu \mathrm{m})$.

\subsection{Integrated $D I C$}

Integrated DIC (i.e., I-DIC) evaluates, for instance, the amplitudes of the displacement fields expressed as Williams' series $[17,18]$. In other words, the kinematic parameterization is tailored to the studied experiment. DIC, and in particular I-DIC, are based on the gray level conservation between two images

$$
I_{0}(\mathbf{x})=I_{t}(\mathbf{x}+\mathbf{u}(\mathbf{x}))
$$

where $I_{0}$ is the picture of the reference configuration, and $I_{t}$ that of the deformed configuration (at time $t$ ). In the absence of noise, $I_{0}$ is related to $I_{t}$ and $\mathbf{u}(\mathbf{x})$, which denotes the displacement for each pixel position $\mathbf{x}$ in the image, by the above equation. The sought displacement field $\mathbf{u}$ will minimize the difference between both terms. It is written as the sum of squared differences over the region of interest (ROI)

$$
\phi=\sum_{R O I}\left(I_{0}(\mathbf{x})-I_{t}(\mathbf{x}+\mathbf{u}(\mathbf{x}))\right)^{2}
$$



tions. For example, a series of mechanically meaningful fields $\boldsymbol{\Psi}_{i}$ (e.g., Williams' series) with unknown amplitudes $v_{i}$ (e.g., SIFs) as the sought parameters

$$
\mathbf{u}(\mathbf{x})=\sum_{i} v_{i} \boldsymbol{\Psi}_{i}(\mathbf{x})
$$

so that $\phi$ will depends on the column vector $\{\boldsymbol{v}\}$ gathering all amplitudes $v_{i}$.

\subsubsection{Williams' series}

Assuming a crack path along the negative $x$-axis, and the crack tip position at the origin, the displacement fields read

$$
\mathbf{u}(\mathbf{z})=\sum_{j=I}^{I I} \sum_{n=p_{i}}^{p_{f}} \omega_{n}^{j} \boldsymbol{\psi}_{n}^{j}(\mathbf{z})
$$

where the vector fields are defined in the complex plane

$$
\mathbf{z}=x+i y=r \exp (i \theta)
$$

with, $j=I$ for a mode I fracture regime

$$
\begin{aligned}
\psi_{n}^{I}(\mathbf{z})=\frac{A(n)}{2 \mu \sqrt{2 \pi}} r^{\frac{n}{2}} & {\left[\kappa \exp \left(\frac{i n \theta}{2}\right)-\frac{n}{2} \exp \left(\frac{i(4-n) \theta}{2}\right)\right.} \\
& \left.+\left((-1)^{n}+\frac{n}{2}\right) \exp \left(-\frac{i n \theta}{2}\right)\right]
\end{aligned}
$$

and $j=I I$ for a mode II regime

$$
\begin{aligned}
\boldsymbol{\psi}_{n}^{I I}(\mathbf{z})=\frac{i A(n)}{2 \mu \sqrt{2 \pi}} r^{\frac{n}{2}} & {\left[\kappa \exp \left(\frac{i n \theta}{2}\right)+\frac{n}{2} \exp \left(\frac{i(4-n) \theta}{2}\right)\right.} \\
& \left.+\left((-1)^{n}-\frac{n}{2}\right) \exp \left(-\frac{i n \theta}{2}\right)\right]
\end{aligned}
$$



the stress intensity factor results were stable when $p_{f} \geq 8$. However, as the present experiments relate to a different class of material (i.e., PMMA), the 
influence of the number of terms will be studied.

Figure 7 displays $K_{I}$ and $c$ (in pixel units) determinations for different positions along the path of the propagating crack (i.e., for different pictures). These results highlight that adding more fields improves the convergence values. In general, $p_{f}=5$ seems sufficient to describe well the experiments in the TDCB specimen of PMMA loaded at $0.5 \mu \mathrm{m} / \mathrm{s}$ CMOD, although in the case of images 330 and 380 the results become stable staring from $p_{f}=7$. It is worth noting that these two images correspond to a few seconds prior to the end of the test, which means that the crack extension is approaching the external boundary and the results become less precise. In the following, all reported results will consider $p_{f}=7$.
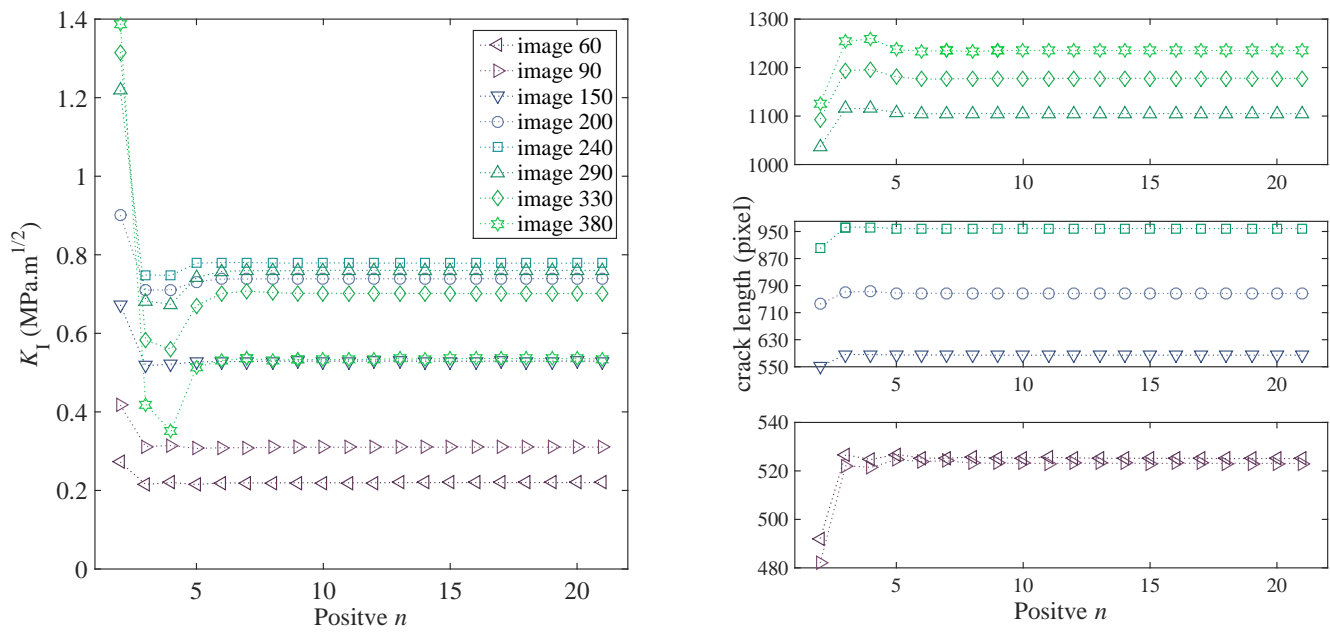

Figure 7: Contribution of the terms of Williams' series for the different image steps along the crack path. Stress intensity factor $K_{I}$ (left) and crack length (right) at different loading phases vs. maximum order $n=p_{f}$ in William's fields. 
image

$$
\eta(\mathbf{x})=I_{0}(\mathbf{x})-I_{t}(\mathbf{x}+\mathbf{u}(\mathbf{x}))
$$

allows for the precise identification of the cracked region [15] since the continuity enforced by triangular meshes is not satisfied across the crack mouth (Figure 8). From this information, the crack path is visible (red dashed 275 line). The latter is used for all I-DIC calculations and the crack tip position along this straight path is then determined by canceling out the amplitude $\omega_{-1}^{I}[33,21]$.

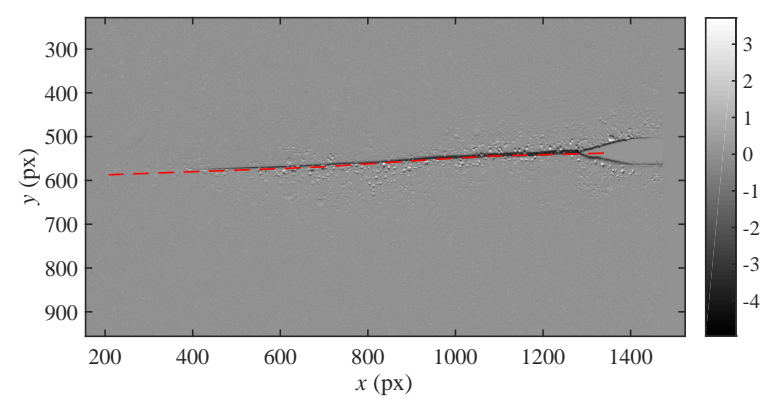

Figure 8: Gray level residual map for the last image revealing the path followed by the crack in the second experiment ( 1 pixel $\equiv 48 \mu \mathrm{m}$ ). The dashed line represents the propagation direction.

Figure 9 shows the displacement fields of the last loading step. There is no clear displacement gradient along the crack direction of the horizontal component. Conversely, there is a strong displacement gradient in the vertical direction. From this qualitative observation, it is expected that the experiment is under dominant mode I condition. This observation will be quantitatively confirmed via I-DIC. 


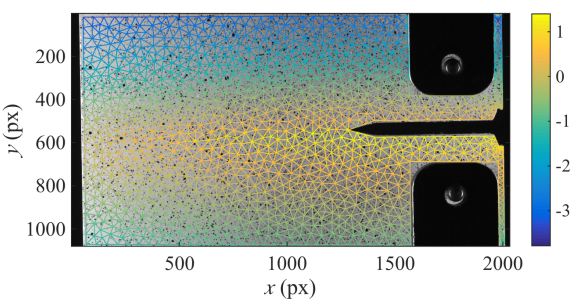

(a)

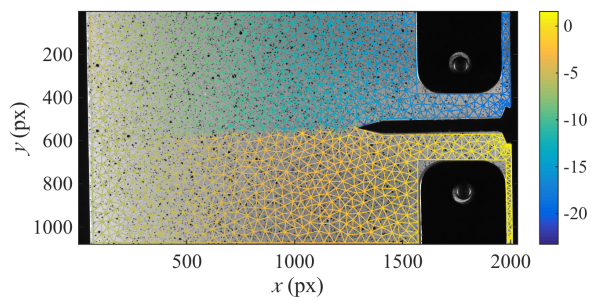

(b)

Figure 9: Displacement fields corresponding to the residual map of Figure 8. Horizontal $u_{x}$ (a) and vertical $u_{y}$ (b) displacement maps obtained from T3-DIC. The values are expressed in pixels $(1$ pixel $\equiv 48 \mu \mathrm{m})$.

\subsubsection{Out-of-plane motions}

For the TDCB tests reported herein, the sample thinness and the use of a single camera made the setup susceptible to out-of-plane sample motions. To account for the presence of out-of-plane motion of the sample, the Williams' series is enriched with additional fields that correct for small rigid body rotations about the horizontal $(x)$ and vertical $(y)$ axes. Any out-of-plane rotation of the sample, in combination with a non-telecentric lens, will be interpreted as in-plane deformation if not considered. Using a pinhole camera model, these apparent displacements can be modeled and a first order approximation returns the following five shape functions [34],

$$
\begin{aligned}
& \boldsymbol{\psi}_{n_{f}+1}=x \mathbf{x}+y \mathbf{y}, \\
& \boldsymbol{\psi}_{n_{f}+2}^{-}=-x^{2} \mathbf{x}-x y \mathbf{y}, \\
& \boldsymbol{\psi}_{n_{f}+2}^{+}=x^{2} \mathbf{x}-x y \mathbf{y}, \\
& \boldsymbol{\psi}_{n_{f}+3}^{-}=-y^{2} \mathbf{y}-x y \mathbf{x}, \\
& \boldsymbol{\psi}_{n_{f}+3}^{+}=y^{2} \mathbf{y}-x y \mathbf{x} .
\end{aligned}
$$


Equation (15) expresses magnification changes due to a translation along the optical axis. Equations (16)-(17) account for the apparent deformation for negative and positive rotations respectively about the image vertical axis. Likewise Equations (18)-(19) express the deformation to account for rotation about the image horizontal direction. Thus, the description of all rigid body motions along the system coordinates of the camera is defined by the five fields introduced above. It is worth recalling that Equation (9), which is truncated between $n=p_{i}$ and $p_{f}$, is solved numerically, to explain that in this particular case these extra modes are added to the truncated series.

\section{Results and discussion}

In this section, both TDCB experiments are studied independently, comparing the fracture properties obtained when using the method based on compliance variations and the integrated DIC framework. Let us first highlight the differences between both test configurations describing them in detail. In the first case, the specimen geometry had its original crack length (36.5 mm) modified by a laser cutting extension accompanied by a pre-load procedure of the sample, which yielded a TDCB specimen with $c_{\text {ini }}=60 \mathrm{~mm}$. Furthermore, the displacements were recorded by a clip gauge that also controlled the fracture experiment at a constant crack mouth opening rate of $5 \mu \mathrm{m} / \mathrm{s}$. In the second experiment no clip gauge was used and no pre-loading was applied. The crack mouth opening displacements $\delta$ were measured via T3-DIC after the test. Figure 5 displays the two ROIs where $\delta$ was measured. Once $\delta$ is obtained from DIC (instead of the clip gauge) and force-time data (i.e., $F(t)$ ) are available, the compliance based method is applied as well. 
It is worth noting that the main differences between both tests are related relationship and is consistent with previous measurements of toughness in PMMA (see for example Ref. [35]). As demonstrated in Section 4.2, I-DIC 
allows the presence (or absence) of mode II regime to be evaluated. It is found that $K_{I I}$ remains very close to zero all along crack propagation. This observation is consistent with the criterion of local symmetry [36, 37, 38, 39] that predicts crack propagation along a path that satisfies $K_{I I}=0$ (here, a straight path perpendicular to the loading axis).
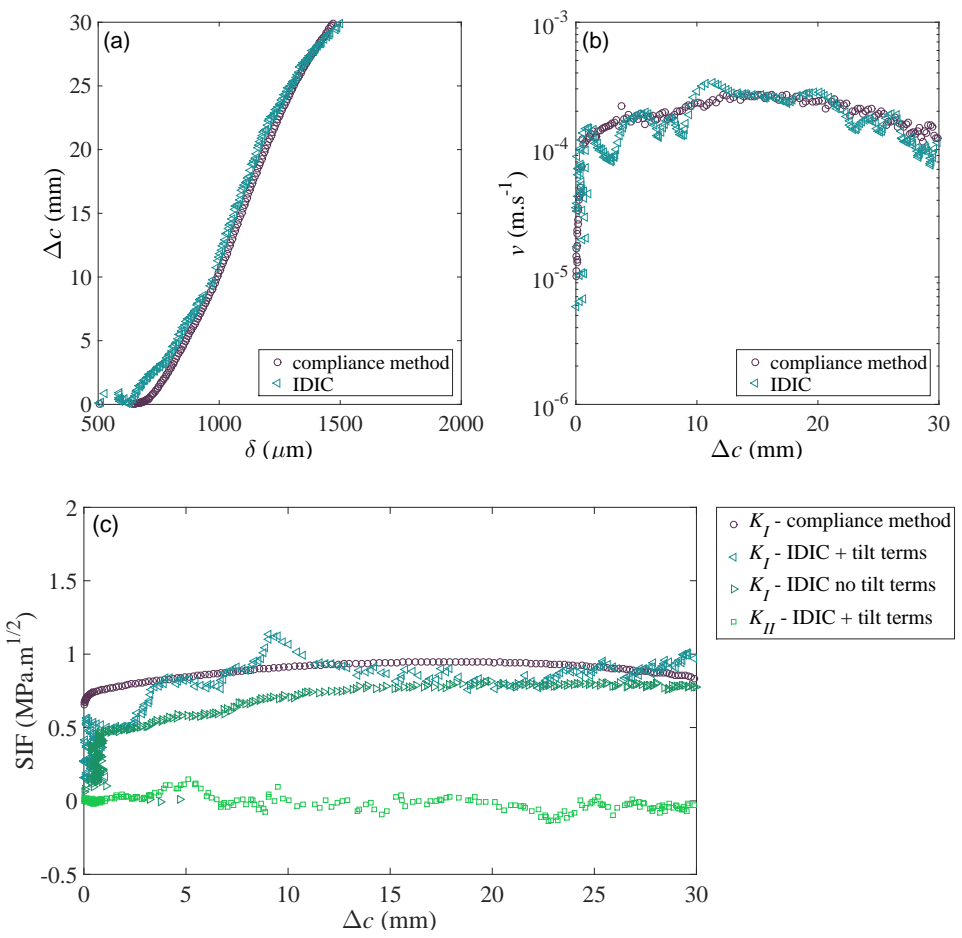

Figure 10: Comparison between the compliance method and the I-DIC technique results for the TDCB specimen tested with clip gauge. (a) Crack length as a function of CMOD, (b) crack velocity as a function of crack length and (c) stress intensity factor as a function of crack length.

\subsection{Second test}

Figure 11 displays crack length, crack velocity, and SIFs for the second experiment (controlled by the stroke speed of $2.5 \mu \mathrm{m} / \mathrm{s}$ ). First, an unstable 
regime of approximately $10 \mathrm{~mm}$ extension is observed when the crack starts to propagate. In this zone, no experimental data were acquired because of the very high speed reached by the propagating crack, which is characterized 

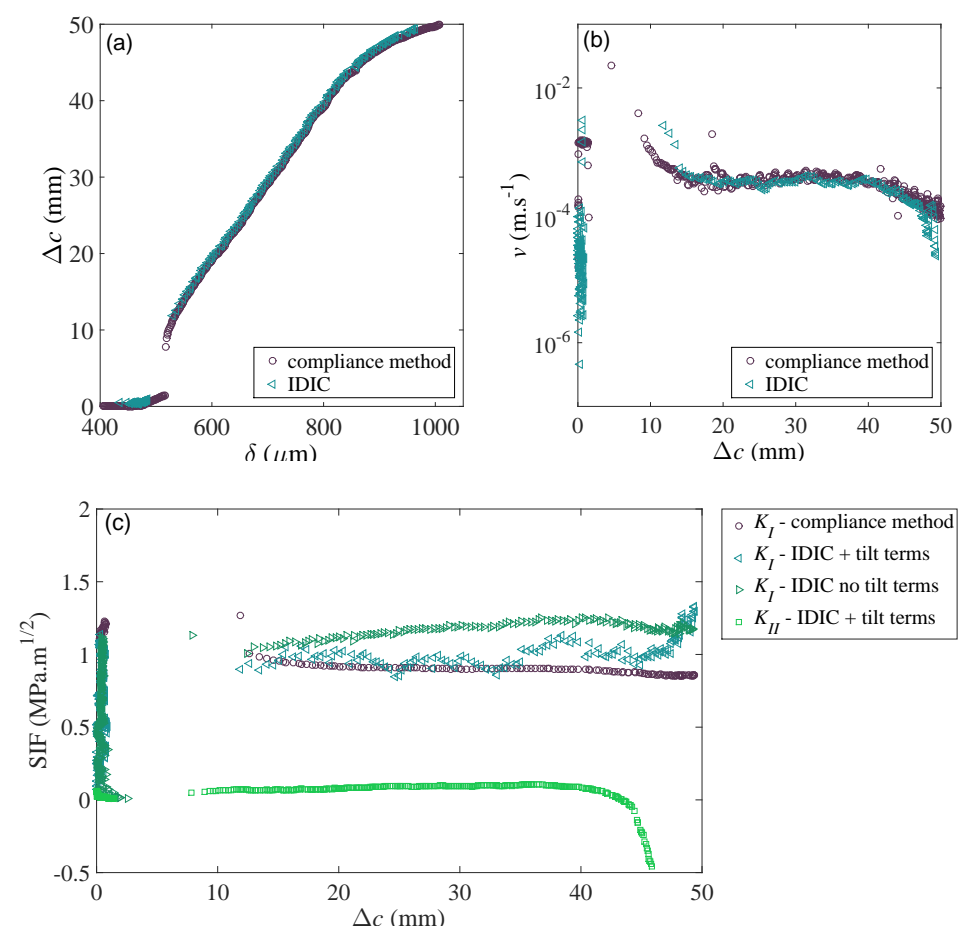

Figure 11: Comparison between the compliance method and the I-DIC technique results for the TDCB specimen tested with no clip gauge. (a) Crack length as a function of CMOD, (b) crack velocity as a function of crack length and (c) stress intensity factor as a function of crack length.

\subsection{Discussion}

Figure 12 shows the changes of the root mean square gray level residuals normalized by the dynamic range of the picture in the reference configuration for the two phases of the test that could be monitored, namely, prior to initiation, and in the stable propagation regime. For instance, during the first phase of the second test, these dimensionless residuals vary from 0.105 and 0.115 with an average value of 0.11 . It is believed that the main reason for these rather high values is due to lighting. In the present case two LED panels 
375 with respect to each other. Minute rotations of the sample changed the brightness of the acquired pictures. For most of the stable crack propagation regime, the residuals remain of the same order as in the first phase. This observation allows the results reported herein to be deemed as trustworthy. It are systematically higher than for the first phase. It is believed that this trend is due to out of plane motions. When these new terms were added to the displacement basis, a small decrease of gray level residuals was observed.
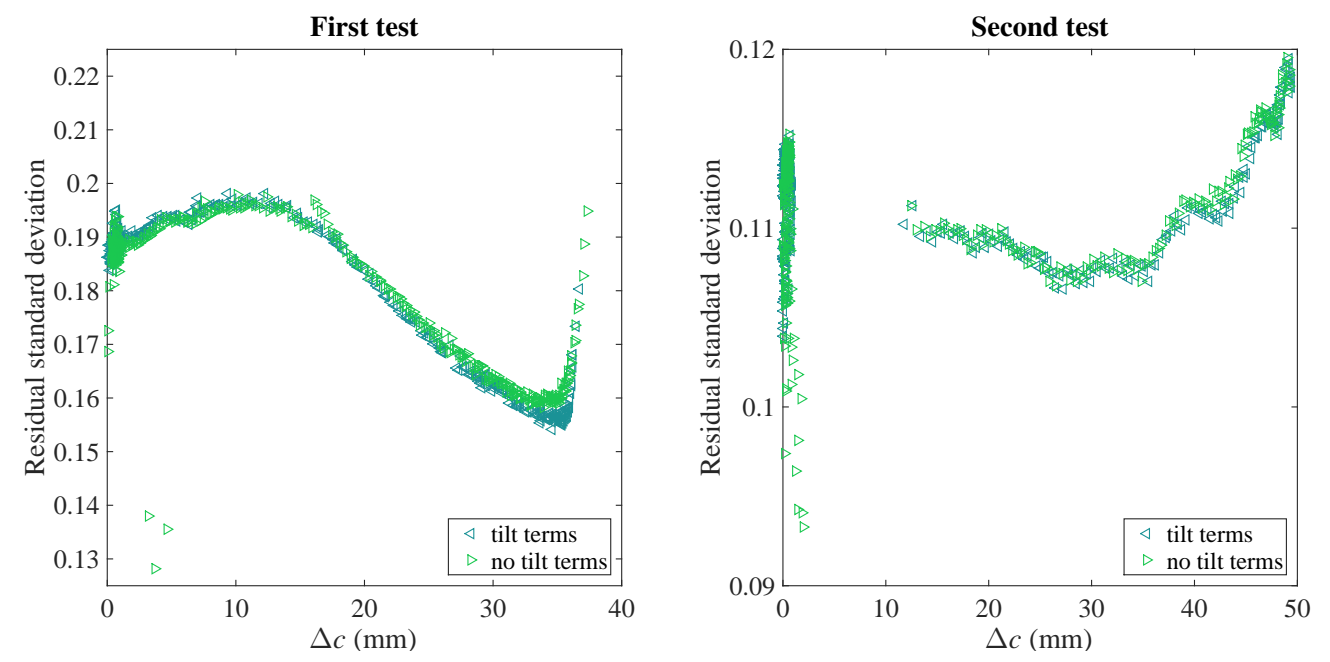

Figure 12: Dimensionless gray level residuals with and without additional displacement fields as function of the crack length for the TDCB specimens tested with clip gauge (left) and with no clip gauge (right).

385

The following discussion compares the evaluations of mode I stress intensity factors with and without the additional displacement fields, namely tilt shape functions. Figure 10(c) shows that the stress intensity factors are more 
consistent with the compliance method when these additional fields are included in the measurement basis. They also lead to slightly lower correlation residuals (see Figure $12($ left $)$ ). In the present case, the $K_{I}$ estimates without the additional fields are essentially biased by an offset of about $0.2 \mathrm{MPa} \sqrt{\mathrm{m}}$ and those with the additional fields yield maximum fluctuations of the same order of magnitude.

For the second test (see Figure 11(c)), $K_{I}$ shows an increasing offset trend starting from $\Delta c=10 \mathrm{~mm}$ up to $\approx 40 \mathrm{~mm}$. Its level is of the order of $0.4 \mathrm{MPa} \sqrt{\mathrm{m}}$ when the additional displacement fields are not included in the kinematic basis. When they are included, the results are more consistent with the compliance method, even though they degrade when $\Delta c>45 \mathrm{~mm}$. This trend is confirmed in both cases by analyzing the dimensionless residuals ${ }_{400}$ (Figure 12(right)). When $\Delta c>30 \mathrm{~mm}$, they increase significantly, thereby indicating that the registrations are less trustworthy, which is consistent with the observations of SIF estimates.

Overall, I-DIC results show some variations of SIF with crack length that are not explained from the mechanical response. For example, considering $\Delta c$ ranging between 5 and $30 \mathrm{~mm}$ for the first test (Figure 10(c)) that corresponds to a plateau of SIF levels according to the compliance method, it is observed that $K_{I}$ fluctuates of about $\pm 0.15 \mathrm{MPam}^{1 / 2}$. In recent works $[32,21]$, such small fluctuations on the SIF and T-stress values were also reported. This particular point would deserve further work. 


\section{Conclusion}

In this work, two tapered double cantilever beam experiments were discussed independently. The results show that the TDCB geometry adopted herein induces stable fracture. In particular, it was highlighted in the second test by its ability of restoring the slow (i.e., stable) crack propagation regime even after being unintentionally introduced to a fast and unstable phenomenon at the initiation stage. All these observations validate the numerical analyses performed to design such tests and confirm that this newly developed fracture test geometry is appropriate to characterize the fracture properties of brittle solids such as PMMA.

${ }_{420}$ Integrated DIC was successfully used to analyze fracture mechanics parameters (e.g., crack tip position, SIFs, mode mixity) of PMMA in experiments using the TDCB geometry. In particular, it was shown that the tests are mode I dominant, which was assumed a priori in the numerical simulations. A comparison study between the I-DIC estimates and those obtained with a method based on compliance variations showed that both approaches were consistent for both investigated tests. To achieve such agreements, it was shown that global displacements describing out-of-plane rigid motions had to be added to Williams' kinematic basis. Another route, which would be worth investigating in the future, would be to consider stereocorrelation techniques to add more freedom to the out-of-plane kinematics [30].

Overall, the integrated DIC analyses show promising results and appear as a reliable method to determine fracture parameters in the TDCB configuration. This paves the way to the characterization of the fracture behavior of materials under extreme conditions where mechanical gauges may be limited 
${ }_{435}$ (e.g., at high temperatures or for fast propagation regimes). Let us note that this technique has already been successfully employed to determine fracture properties of other brittle $[18,41,21]$ or ductile $[20,16,33,32]$ materials. One additional result of the present work was its validation against an independent method.

${ }_{440}$ Acknowledgments

It is a pleasure to acknowledge the financial support of the Brazilian agencies $\mathrm{CNPq}$ and CAPES to this investigation during the doctoral thesis and postdoctoral study of TMG. TMG's stay at UPMC and LMT was supported by the Doctoral Sandwich Program Abroad (PDSE fellowship) of CAPES, 445 grant \#99999.009821/2014 - 07. This work was also partially supported by BPI France within the DICCIT project. 


\section{References}

[1] ASTM E1820-17, Standard Test Method for Measurement of Fracture Toughness, ASTM International, West Conshohocken, PA (USA), 2017.

[2] A. Shyam, E. Lara-Curzio, The double-torsion testing technique for determination of fracture toughness and slow crack growth behavior of materials: A review, Journal of Materials Science 41 (13) (2006) 40934104 .

[3] H. Liebowitz, Fracture, vol. 5 Edition, Academic, New York, 1969.

[4] T. M. Grabois, Experimental fracture mechanics of cement-based materials: A new methodology for the accurate measurement of the material toughness, PhD thesis, Universidade Federal do Rio de Janeiro (2016).

[5] A. V. Vasudevan, T. M. Grabois, G. C. Cordeiro, R. D. Toledo Filho, L. Ponson, A new fracture test methodology for the accurate characterization of brittle fracture properties, (in preparation).

[6] J. P. Gallagher, Experimentally determined stress intensity factors for several contoured double cantilever beam specimens, Engineering Fracture Mechanics 3 (1) (1971) 27-43.

[7] H. L. Marcus, G. C. Sih, A crackline-loaded edge-crack stress corrosion specimen, Engineering Fracture Mechanics 3 (4) (1971) 453-461.

[8] J. F. Davalos, P. Madabhusi-Raman, P. Z. Qiao, M. P. Wolcott, Compliance rate change of tapered double cantilever beam specimen with hy- 
brid interface bonds, Theoretical and Applied Fracture Mechanics 29 (2) (1998) 125-139.

470

[14] P. Forquin, L. Rota, Y. Charles, F. Hild, A method to determine the toughness scatter of brittle materials, International Journal of Fracture 125 (1) (2004) 171-187. 
[15] S. Roux, J. Réthoré, F. Hild, Digital Image Correlation and Fracture: An Advanced Technique for Estimating Stress Intensity Factors of 2D and 3D Cracks, Journal of Physics D: Applied Physics 42 (2009) 214004.

[16] F. Mathieu, F. Hild, S. Roux, Identification of a crack propagation law by digital image correlation, International Journal of Fatigue 36 (1) (2012) 146-154.

[17] M. L. Williams, On the stress distribution at the base of a stationary crack, Journal of Applied Mechanics 24 (1) (1957) 109-114.

[18] S. Roux, F. Hild, Stress intensity factor measurements from digital image correlation: post-processing and integrated approaches, International Journal of Fracture 140 (1-4) (2006) 141-157.

[19] M. Y. Dehnavi, S. Khaleghian, A. Emami, M. Tehrani, N. Soltani, Utilizing digital image correlation to determine stress intensity factors, Polymer Testing 37 (2014) 28-35.

[20] R. Hamam, F. Hild, S. Roux, Stress intensity factor gauging by digital image correlation: Application in cyclic fatigue, Strain 43 (2007) 181192.

[21] R. Vargas, J. Neggers, R. Canto, J. Rodrigues, F. Hild, Analysis of wedge splitting test on refractory castable via integrated DIC, Journal of the European Ceramic Society 36 (16) (2016) 4309-4317.

[22] S. Lampman, Characterization and failure analyses of plastics, ASM International, Materials Park, Ohio, 2003. 
[23] H. Leclerc, J. Périé, S. Roux, F. Hild, Integrated digital image correlation for the identification of mechanical properties, Vol. LNCS 5496, Springer, Berlin (Germany), 2009, pp. 161-171.

515

[24] S. Morel, G. Mourot, J. Schmittbuhl, Influence of the specimen geometry on R-curve behavior and roughening of fracture surfaces, International Journal of Fracture 121 (2003) 23-42.

[25] CAST3M, Finite Element package developed by CEA, France: http://www-cast3m.cea.fr/index.php (version 2015).

[26] M. Kanninen, C. Popelar, Advanced Fracture Mechanics, Oxford University Press, Oxford (UK), 1985.

[27] B. Lawn, Fracture of brittle solids, 2nd Edition, Cambridge University Press, Cambridge, 1993.

[28] T. Chu, W. Ranson, M. Sutton, Applications of digital-image-correlation techniques to experimental mechanics, Experimental Mechanics 25 (3) (1985) 232-244.

[29] M. Sutton, C. Mingqi, W. Peters, Y. Chao, S. McNeill, Application of an optimized digital correlation method to planar deformation analysis, Image and Vision Computing 4 (3) (1986) 143-150.

[30] M. Sutton, Computer vision-based, noncontacting deformation measurements in mechanics: A generational transformation, Applied Mechanics Reviews 65 (AMR-13-1009, 050802). 
[31] C. Henninger, S. Roux, F. Hild, Enriched kinematic fields of cracked structures, International Journal of Solids and Structures 47 (24) (2010) $3305-3316$.

[32] J. Réthoré, Automatic crack tip detection and stress intensity factors estimation of curved cracks from digital images, International Journal for Numerical Methods in Engineering 103 (7) (2015) 516-534.

[33] F. Mathieu, F. Hild, S. Roux, Image-based identification procedure of a crack propagation law, Engineering Fracture Mechanics 103 (2013) $48-59$.

[34] J. Le Flohic, V. Parpoil, S. Bouissou, M. Poncelet, H. Leclerc, A 3D Displacement Control by Digital Image Correlation for the Multiaxial Testing of Materials with a Stewart Platform, Experimental Mechanics 54 (5) (2014) 817-828.

[35] G. P. Marshall, L. H. Coutts, J. G. Williams, Temperature effects in the fracture of PMMA, Journal of Material Science 9 (1974) 1409-1419.

[36] R. V. Gol'dstein, R. L. Salganik, Brittle fracture of solids with arbitrary cracks, International Journal of Fracture 10 (1974) 507-523.

[37] M. Amestoy, H. Bui, K. Dang Van, Déviation infinitésimale d'une fissure dans une direction arbitraire, Comptes Rendus de l'Académie des Sciences Paris t. 289 (Série B) (1979) 99-103.

[38] B. Cotterell, J. R. Rice, Slightly curved or kinked cracks, International Journal of Fracture 16 (1980) 155-169. 
555 [39] M. Amestoy, H. Bui, K. Dang Van, Analytic asymptotic solution of the kinked crack problem, in: D. François (Ed.), Proceedings of ICF5, Vol. 1, Pergamon Press, 1981, pp. 107-113.

[40] K. Ravi-Chandar, M. Balzano, On the mechanics and mechanisms of crack growth in polymeric materials, Engineering Fracture Mechanics 30 (5) (1988) 713-727.

[41] J. Réthoré, S. Roux, F. Hild, An extended and integrated digital image correlation technique applied to the analysis of fractured samples. The equilibrium gap method as a mechanical filter, European Journal of Computational Mechanics 18 (3-4) (2009) 285-306. 